\title{
What affects the Speed of a Car? Let's Investigate!
}

\author{
Sondos Mohammad Hamideh ${ }^{1}$ \\ ${ }^{1}$ Journal of Conceptual and Applied STEM Education
}

July 29, 2020

\section{Introduction}

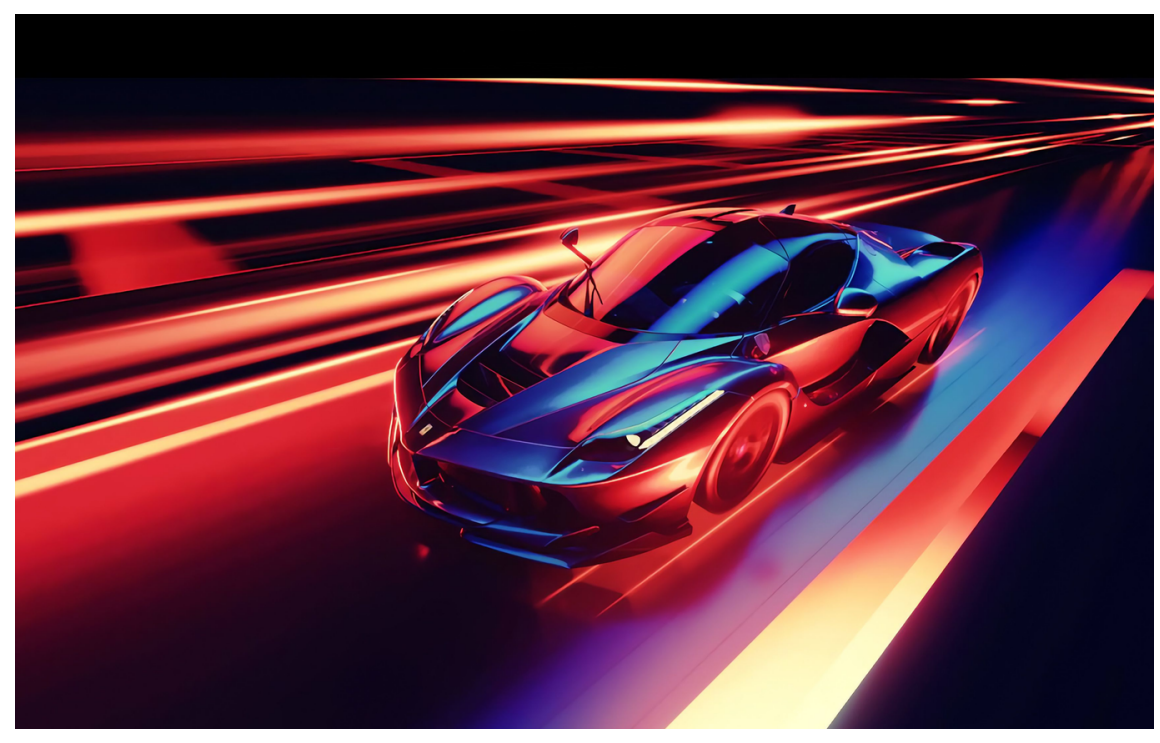

Figure 1: Speed car project

Over the years, Engineers have been working on improving the key factors that determines the quality when manufacturing cars that are mostly used in racing contests. As the journey of these tests have not ended yet, it was agreed on some of the main factors that affects the performance of a race car. In this lesson, students will be those engineers that will get the chance to hold several tests and record their findings on a simple car model made from the LEGO Education robotics toolbox WeDo 2.0!

\section{Lesson Objectives}

1. Students will be able to describe the importance of Engineers role at car's manufacturing factories.

2. Students will be able to form a definition for the physical term Speed in relation to car's performance.

3. Students will be able to hold a test to determine the impact of several elements in a car's performance and present it to the class. 


\section{Materials}

- LEGO Education WeDo 2.0 kit

- Laptop / Tablet that has the WeDo 2.0 Software

- Papers, Paper markers, Coloring pens, sticky tape

- Student worksheet to print, https://drive.google.com/file/d/ 1Nn9vUGe4lw8PE05cUx1ZCrfpiW47ITHX/view?usp=sharing

- Metric printable ruler, http://www.vendian.org/mncharity/dir3/paper_rulers/UnstableURL/ rulers_metric.pdf

\section{Vocabulary}

- Speed

- Acceleration

- Pulleys as Mechanical configuration

\section{Media Resources}

- Video," Explore Video - Speed - WeDo 2.0 - LEGO Education", https://youtu.be/5vbvRw-hUGO

- Article," What makes a car faster?" https://sciencing.com/car-fast-7564824.html

- Article, "Auto Racing" https://en.wikipedia.org/wiki/Auto_racing\#Racing-car_setup

\section{Lesson Plan}

\section{Pre lesson preparation:}

- Divide students into groups (each consists of 3) and place and stick on each table the metric ruler so that students can use it as a reference at the test, and highlight a starting point.

- Place a LEGO Education WeDo 2.0 kit on each table with a laptop/tablet, along with one Student worksheet per group and some extra papers, pens.

- Ask the students to read the article "Auto Racing" before the class time.

\section{In class lesson steps:}

- Start your lesson as shown in the Teachers manual for the speed lesson by LEGO education by playing the Video at first and then discuss the idea of the racing cars that are used in contests as following:

1. Auto racing (also known as car racing, motor racing, or automobile racing) is a motorsport involving the racing of automobiles for competition .In auto racing, the racing setup or car setup is the set of adjustments made to the vehicle to optimize its behaviour (performance, handling, reliability, etc.). Adjustments can occur in suspensions, brakes, transmissions, engines, tires and other factors.

2. Speed is the rate of motion, or the rate of change of position. It is expressed as distance moved (d) per unit of time(t). As in SI units, it's meters/second.

3. Acceleration is defined as the rate of change of the speed. It is thus a vector quantity with dimension length/time ${ }^{2}$. In SI units, acceleration is measured in meters $/$ second $^{2}$.

- Introduce the lesson for the students as they are Engineers in a car manufacturing factory and their mission today is to report what are the changes that we can do to make the car model prototype made 
from the LEGO Education kit go faster. Inform the students that in this lesson they will be given a 15 minutes to build the car instructed in the software( From the Guided projects menu -Speed and skipping directly to the Build phase, and inform students to ignore adding the motion sensor as it will not be used ) and then ask all students to program as following so that the electrical features are all the same and only the mechanical configurations will be adjusted during the test:

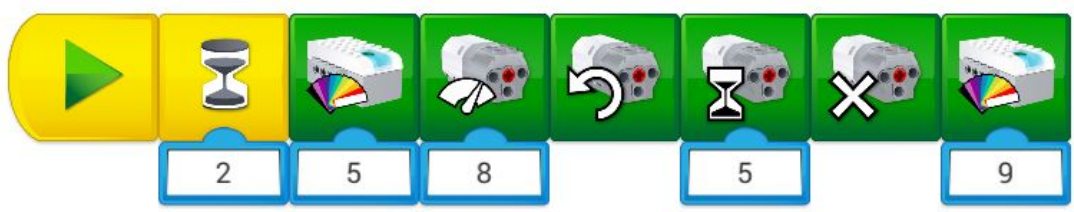

Figure 2: Speed car model program

- Ask students to place the car model at the pointed reference point. In this step students will record the effect of changing the wheel diameter on the speed. Using the different wheel pieces given in the kit they should change them and test each one and fill in the worksheet how much distance (based on the metric ruler paper they should make calculations) did the car record on each set. At last they should chose one configuration to be their optimum choice.

- Students shall be considering changing the Pulley system configuration that has been made in the model. A pulley is a simple machine that can be used to lift objects and transmit motion from a mechanical part to another. In this model, the electrical motion from the Motor is linked through the rounded blue pulley attached to the motor and then transmitted to the wheels through a rubber band. Students now should test the three pulley configurations demonstrated in the worksheet and record the covered distance to then choose an optimal solution.

- One final optional step is that giving the students around 15 minutes to adjust the shape of the car to illustrate the effect of the Weight on the aerodynamics of the car. Several adjustments shall be done until the car breaks the last record of distance covered at the previous step.

- Ask the students to prepare a 3 minutes presentation to reflect on the test they have made with their classmates. Make sure all groups share their findings.

- At last, adjust your classroom configuration to host a race using the final prototypes made from each group and announce the winner based on the correct suitable adjustments they have made.

\section{Lesson Assessment}

1. Collect the worksheets the students have filled to use as a reference for the grading marks you plan to decided.

2. Randomly number each car model and ask students to vote for the best final design. 